Andreas Charidimou,

$\mathrm{MD}, \mathrm{MSc}$

Andre Philippe Peeters, MD

Rolf Jäger, MD

Zoe Fox, PhD

Yves Vandermeeren, MD, $\mathrm{PhD}$

Patrice Laloux, MD, PhD Jean-Claude Baron, PhD

David John Werring, $\mathrm{PhD}$

Correspondence to Dr. Werring: d.werring@ucl.ac.uk

Supplemental data at www.neurology.org

\section{Cortical superficial siderosis and intracerebral hemorrhage risk in cerebral amyloid angiopathy}

ABSTRACT

Objective: To investigate whether cortical superficial siderosis (cSS) on MRI, especially if disseminated (involving more than 3 sulci), increases the risk of future symptomatic lobar intracerebral hemorrhage $(\mathrm{ICH})$ in cerebral amyloid angiopathy (CAA).

Methods: European multicenter cohort study of 118 patients with CAA (104 with baseline symptomatic lobar $\mathrm{ICH}$ ) diagnosed according to the Boston criteria. We obtained baseline clinical, MRI, and follow-up data on symptomatic lobar $\mathrm{ICH}$. Using Kaplan-Meier and Cox regression analyses, we investigated cSS and ICH risk, adjusting for known confounders.

Results: During a median follow-up time of 24 months (interquartile range 9-44 months), 23 of 118 patients (19.5\%, 95\% confidence interval [CI]: 12.8\%-27.8\%) experienced symptomatic lobar ICH. Any cSS and disseminated cSS were predictors of time until first or recurrent ICH (logrank test: $p=0.0045$ and $p=0.0009$, respectively). $\mathrm{ICH}$ risk at 4 years was $25 \%(95 \% \mathrm{Cl}$ : 7.6\%-28.3\%) for patients without siderosis; $28.9 \%$ (95\% Cl: $7.7 \%-76.7 \%)$ for patients with focal siderosis; and 74\% (95\% Cl: 44.1\%-95.7\%) for patients with disseminated cSS (log-rank test: $p=0.0031$ ). In Cox regression models, any cSS and disseminated cSS were both independently associated with increased lobar ICH risk, after adjusting for $\geq 2$ microbleeds and age (hazard ratio: $2.53 ; 95 \% \mathrm{Cl}: 1.05-6.15 ; p=0.040$ and hazard ratio: 3.16 ; $95 \% \mathrm{Cl}: 1.35-$ 7.43; $p=0.008$, respectively). These results remained consistent in sensitivity analyses including only patients with symptomatic lobar $\mathrm{ICH}$ at baseline.

Conclusions: Our findings indicate that cSS, particularly if disseminated, is associated with an increased risk of symptomatic lobar ICH in CAA. cSS may help stratify future bleeding risk in CAA, with implications for prognosis and treatment. Neurology ${ }^{\circledR} 2013 ; 81: 1666-1673$

\section{GLOSSARY}

CAA = cerebral amyloid angiopathy; $\mathbf{C l}=$ confidence interval; $\mathbf{C M B}=$ cerebral microbleed; $\mathbf{c S S}=$ cortical superficial siderosis; FLAIR = fluid-attenuated inversion recovery; $\mathbf{G R E}=$ gradient-recalled echo; $\mathbf{H R}=$ hazard ratio; $\mathbf{I C H}=$ intracerebral hemorrhage.

Sporadic cerebral amyloid angiopathy (CAA) is a highly prevalent, age-related small-vessel disease $^{1}$ caused by amyloid- $\beta$ deposition in cortical and leptomeningeal vessel walls. ${ }^{2}$ CAA is a major cause of lobar intracerebral hemorrhage $(\mathrm{ICH})$, particularly in elderly patients. ${ }^{2-5}$ Spontaneous ICH is one of the most catastrophic forms of stroke, with a high risk of recurrence ${ }^{6-8}$; CAA-related lobar ICH may carry a greater risk than deep ICH presumed to be due to hypertensive arteriopathy, ${ }^{8,9}$ but this is currently difficult to predict.

Predisposing factors for lobar ICH and lobar ICH recurrence in CAA include APOE \&4 and $\varepsilon 2$ alleles, ${ }^{10}$ hemorrhagic neuroimaging markers of CAA such as lobar cerebral microbleeds $(\mathrm{CMBs}),{ }^{11}$ and anticoagulant or antiplatelet use. ${ }^{12}$ Little is known about cortical superficial siderosis (cSS), a recently identified neuroimaging marker of CAA, ${ }^{13}$ and the risk of subsequent

From the Stroke Research Group, Department of Brain Repair and Rehabilitation (A.C., D.J.W.), and Lysholm Department of Neuroradiology (R.J.), UCL Institute of Neurology and National Hospital for Neurology and Neurosurgery, Queen Square, London, UK; Department of Neurology (A.P.P.), Cliniques Universitaires UCL Saint Luc, Brussels, Belgium; Department of Brain Repair and Rehabilitation (R.J.), and Biomedical Research Centre, UCL and the Education Unit (Z.F.), UCL Institute of Neurology, Queen Square, London, UK; Department of Neurology, CHU Mont-Godinne (Y.V., P.L.), and Institute of Neuroscience (Y.V., P.L.), Université Catholique de Louvain, Brussels, Belgium; Department of Clinical Neurosciences (J.-C.B.), University of Cambridge, Addenbrooke's Hospital, Cambridge, UK; and UMR 894 INSERMUniversité Paris 5, Sorbonne Paris Cité, Paris, France.

Go to Neurology.org for full disclosures. Funding information and disclosures deemed relevant by the authors, if any, are provided at the end of the article. 
ICH. cSS reflects linear blood residues in the superficial (subpial) layers of the cerebral cortex. ${ }^{14}$ One likely mechanism leading to cSS is repeated episodes of hemorrhage into the subarachnoid space from brittle superficial cortical or leptomeningeal CAA-laden vessels, potentially heralding a high risk of future lobar $\mathrm{ICH}$. A recent study showed that nearly $50 \%$ of CAA patients with cSS experienced intracranial hemorrhage over a period of 35 months, ${ }^{15}$ but this study did not include patients without cSS as a control group.

We tested the hypothesis that in patients with CAA, cSS, especially involving multiple sulci (reflecting more widespread or active disease), is associated with an increased risk of future symptomatic lobar ICH in a European multicenter cohort study.

METHODS Study population and baseline data collection. We included consecutive patients diagnosed with CAA (according to the original Boston criteria, ${ }^{16}$ i.e., not including $\mathrm{CSS}$ as a criterion) at 4 stroke centers over defined time periods. The centers included University College London Hospitals NHS Foundation Trust (London) (March 2003 to September 2011), Addenbrooke's Hospital (Cambridge) (July 2002 to March 2010), Cliniques Universitaires Saint Luc (Brussels) (December 2003 to April 2010), and CHU Mont-

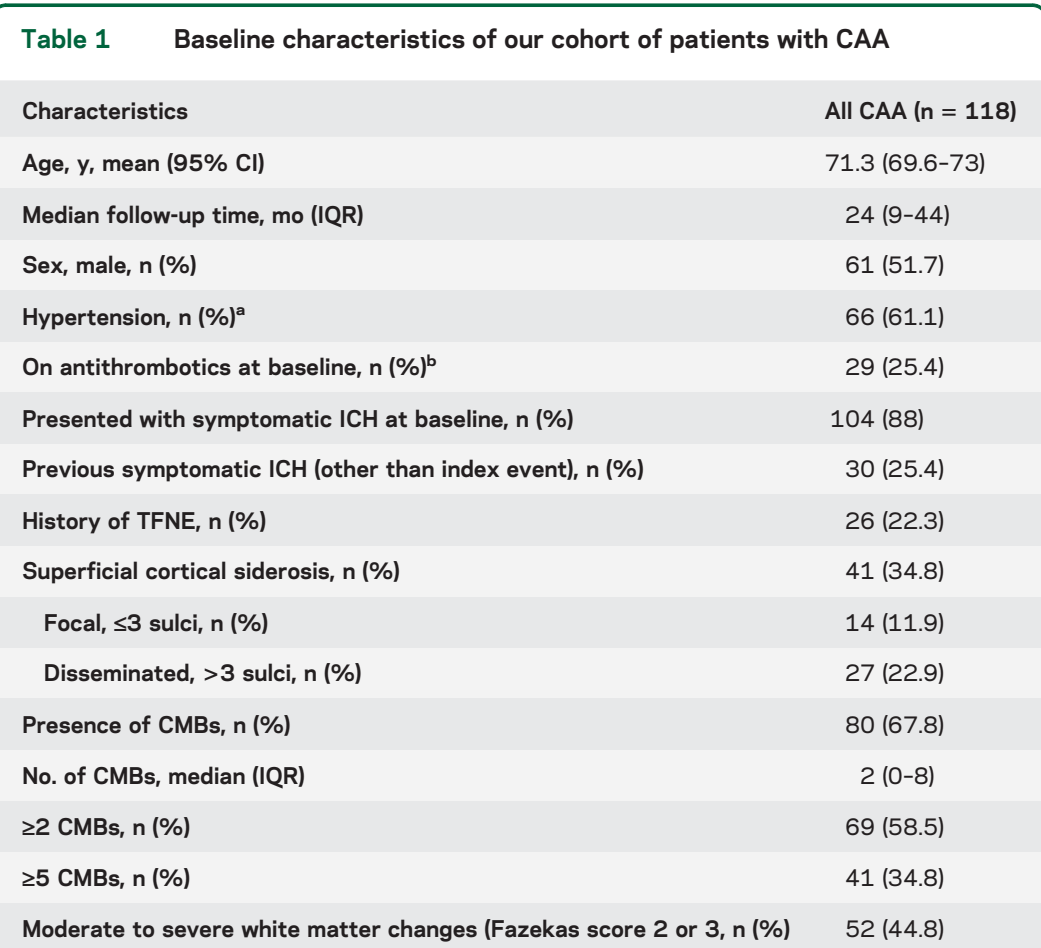

Abbreviations: $\mathrm{CAA}=$ cerebral amyloid angiopathy $; \mathrm{Cl}=$ confidence interval; $\mathrm{CMB}=$ cerebral microbleed; ICH = intracerebral hemorrhage; IQR = interquartile range; TFNE $=$ transient focal neurologic episodes.

${ }^{a}$ Ten patients with missing data.

${ }^{\mathrm{b}}$ Four patients with missing data.
Godinne UCL (August 2005 to March 2009). At participating centers, MRI scanning is a routine investigation for cases of suspected CAA, unless there are contraindications. Our inclusion criteria were 1) patients fulfilling the original Boston criteria for CAA, ${ }^{16}$ 2) available MRI sequences including $\mathrm{T} 2^{*}$-weighted gradient-recalled echo $\left(\mathrm{T} 2{ }^{*}\right.$ GRE) and fluid-attenuated inversion recovery (FLAIR) MRI, and 3) available follow-up information on symptomatic $\mathrm{ICH}$, confirmed by neuroimaging. We included all patients with CAA, including survivors of spontaneous lobar ICH and those who, during investigation for other symptoms, were found to have strictly lobar CMBs (or asymptomatic lobar ICH). We excluded CAA patients without adequate MRI $(\mathrm{n}=26)$ or reliable follow-up data $(\mathrm{n}=37)$. Excluded subjects $(\mathrm{n}=63)$ did not differ significantly from those included in any baseline characteristics (all $p>0.05$ ).

Clinical data at the time of presentation (age, sex, vascular risk factors including hypertension, use of antithrombotics, and previous symptomatic $\mathrm{ICH}$ ) were obtained from prospective databases and by medical records review using standardized data collection forms. A clearly documented history of transient ( $\leq 24$ hours) focal neurologic episodes with no known alternative explanation other than CAA (e.g., structural brain lesion, atrial fibrillation, extracranial or intracranial stenosis) was ascertained by review of medical records.

Standard protocol approvals, registrations, and patient consents. The study received ethical approval by the National Hospital for Neurology and Neurosurgery and Institute of Neurology Joint Research Ethics Committee, the Commission d'Ethique Biomedicale Hospitalo Facultaire of the Faculte de Medicine (Université Catholique de Louvain), and the Comite d'ethique medicale of the CHU Mont-Godinne UCL.

MRI acquisition and analysis. The MRI protocol was similar in each hospital. Imaging was at $1.5 \mathrm{~T}$ field strength for all patients and included T1-weighted, T2-weighted, FLAIR, and axial T2*GRE (slice thickness $5 \mathrm{~mm}$, repetition time 500-1,000 milliseconds, echo time 15-70 milliseconds). Images were reviewed by a trained clinical research fellow blinded to clinical and followup data. The presence and distribution of $\mathrm{CMBs}$ were evaluated on T2*-GRE images using the Microbleed Anatomical Rating Scale. ${ }^{17}$ Asymptomatic or symptomatic prior ICH (>5 mm in diameter on T2*-GRE MRI) was also noted. ${ }^{18} \mathrm{cSS}$ was defined as linear residues of chronic blood products in the superficial layers of the cerebral cortex showing a characteristic "gyriform" pattern of low signal on T2*-GRE images, without corresponding hyperintense signal on T1-weighted or FLAIR images (i.e., without acute subarachnoid hemorrhage). We did not include cSS contiguous with any $\mathrm{ICH}$. The distribution of cSS was classified as focal (restricted to $\leq 3$ sulci) or disseminated $\left(>3\right.$ sulci). ${ }^{13}$ White matter changes were evaluated using the 4-step simplified Fazekas rating scale $(0-3: 0=$ no lesions; $1=$ focal lesions; $2=$ early confluent; 3 = confluent). ${ }^{19}$

Follow-up. Follow-up data were obtained from a systematic review of multiple overlapping sources including prospective databases, medical records review (including discharge summaries, follow-up outpatient and general practitioner letters), and radiologic databases, using standardized data collection forms. We collected information on clinically symptomatic $\mathrm{ICH}$, defined as a symptomatic stroke syndrome associated with neuroimaging evidence of a corresponding ICH ( $>5 \mathrm{~mm}$ in diameter),${ }^{20}$ and death of any cause. Outcome events were assessed using all clinical, radiologic, and pathologic information available, blinded to the presence of cSS at baseline MRI. We determined whether the location of symptomatic ICH at follow-up corresponded to the anatomical distribution of cSS on baseline MRI. 
Statistical analysis. We compared clinical and imaging characteristics of CAA patients with symptomatic lobar ICH during follow-up to patients without $\mathrm{ICH}$, using $\chi^{2}$ tests and the Fisher exact test for categorical variables, and 2-sample $t$ tests or MannWhitney $U$ tests depending on the distribution of continuous variables. The reliability of rating for presence and category rating of cSS was assessed in a sample of MRI scans from patients with probable CAA $(n=48)$ by calculating Cohen $\kappa$ and weighted $\kappa$ statistic, respectively. We also compared basic clinical and imaging characteristics of patients with vs without cSS. We determined the presence of cSS and disseminated cSS as univariate predictors of ICH risk using Kaplan-Meier plots with significance testing by the log-rank test. Survival time was calculated from date of baseline MRI scan until the date of symptomatic lobar $\mathrm{ICH}$ at follow-up or the last known date without the outcome event of interest. For individuals experiencing multiple lobar ICHs during follow-up, data were censored at time of first $\mathrm{ICH}$. Data were also censored at the time of death from causes other than documented symptomatic ICH. Cox regression analysis was performed to calculate univariate hazard ratio (HR) as a measure of the effect size.

We estimated that our study would have a power of $88 \%$ to detect a difference in ICH risk between CAA patients with and without cSS, assuming $50 \%$ and $20 \%$ rates of $\mathrm{ICH}$ over 4 years, respectively ${ }^{11,12,15}$ (2-tailed test and with $\alpha$ values of 0.05 ).

According to the "rule of 10 " for developing proportional hazards models, we require approximately 10 outcome events for each potential covariate in multivariable analyses..$^{21,22}$
Therefore, we investigated the effect of the following prespecified potential predictors using a Cox proportional hazards model: presence of cSS or disseminated cSS, the presence of multiple CMBs $(\geq 2)$, prespecified on the basis of the hypothesized effect on ICH risk from previous published series in CAA, ${ }^{11,12}$ and age, which is an important potential confounder. As sensitivity analyses, we repeated all statistical tests in CAA patients with symptomatic lobar ICH at baseline, and those who had their MRI within 6 months of the index ICH. We also undertook exploratory multivariable models incorporating previous ICH as another potential confounding factor. The proportional hazard assumption was tested using graphical checks and Schoenfeld residualsbased tests.

A $p$ value $\leq 0.05$ was considered to be statistically significant. All analyses were performed using STATA (version 11.2; StataCorp, College Station, TX). This report was prepared with reference to the STROBE (Strengthening the Reporting of Observational Studies in Epidemiology) guidelines. ${ }^{23}$

RESULTS The final cohort consisted of 118 patients fulfilling the Boston diagnostic criteria ${ }^{16}$ for CAA (table 1): 8 with pathologically proven CAA, 86 with probable CAA, and 24 with possible CAA. Of these, 104 patients $(88 \%)$ presented with symptomatic lobar ICH at baseline, 8 with transient focal neurologic episodes, 3 with cognitive decline, 1 with acute convexity subarachnoid hemorrhage, and 2 with

Table 2 Characteristics and comparison of patients with CAA according to symptomatic lobar intracerebral hemorrhage at follow-up

\begin{tabular}{|c|c|c|c|}
\hline Characteristics & $\begin{array}{l}\text { No ICH at follow-up } \\
(n=95)\end{array}$ & $\begin{array}{l}\text { ICH at follow-up } \\
(n=23)\end{array}$ & $p$ Value \\
\hline Age, y, mean $(95 \% \mathrm{Cl})$ & $70.4(68.5-72.3)$ & $75.2(71.5-78.9)$ & $0.026^{a}$ \\
\hline Median follow-up time, mo (IQR) & $24.6(9-51.3)$ & $21.3(6-38.5)$ & 0.429 \\
\hline Sex, male, $n$ (\%) & $51(53.7)$ & $10(43.5)$ & 0.379 \\
\hline Hypertension, $\mathrm{n}(\%)$ & $54(63.5)^{c}$ & 12 (52.2) & 0.322 \\
\hline On antithrombotics at baseline, $n(\%)$ & $23(25.3)^{d}$ & $6(26.1)$ & 0.936 \\
\hline Presented with symptomatic ICH at baseline, $\mathrm{n}(\%)$ & $84(88.4)$ & $20(87)$ & 1.000 \\
\hline Previous symptomatic ICH (other than index event), $n$ (\%) & $23(24.1)$ & $7(30.4)$ & 0.538 \\
\hline History of TFNE, $n(\%)$ & $17(17.9)$ & 9 (39.1) & $0.027^{a}$ \\
\hline Superficial cortical siderosis, $n$ (\%) & $27(28.4)$ & $14(60.9)$ & $0.003^{b}$ \\
\hline Focal, $\leq 3$ sulci, $n$ (\%) & 11 (11.6) & $3(13)$ & 1.000 \\
\hline Disseminated, $>3$ sulci, $n$ (\%) & $16(16.8)$ & $11(47.8)$ & $0.002^{b}$ \\
\hline Presence of CMBs, $n(\%)$ & $62(65.3)$ & $18(78.3)$ & 0.231 \\
\hline No. of CMBs, median (IQR) & $2(0-8)$ & $3(1-15)$ & 0.412 \\
\hline$\geq 2$ CMBs, n (\%) & $54(56.8)$ & $15(65.2)$ & 0.465 \\
\hline$\geq 5$ CMBs, n (\%) & 32 (33.7) & $9(39.1)$ & 0.623 \\
\hline Moderate to severe white matter changes (Fazekas score 2 or 3 ), $n(\%)$ & $41(44.1)$ & $11(47.8)$ & 0.747 \\
\hline
\end{tabular}

Abbreviations: $\mathrm{CAA}=$ cerebral amyloid angiopathy; $\mathrm{Cl}=$ confidence interval; $\mathrm{CMB}=$ cerebral microbleed; $\mathrm{ICH}=$ intracerebral hemorrhage; IQR = interquartile range; TFNE = transient focal neurologic episodes.

The $p$ values refer to differences between patients with vs without symptomatic lobar intracerebral hemorrhage at followup. The median time between all baseline symptomatic ICH and first MRI was 17 days (IQR: 3-75.5 days).

a Statistically significant difference at $p<0.05$.

${ }^{b}$ Statistically significant difference at $p<0.01$.

${ }^{\mathrm{c}}$ Ten patients with missing data.

${ }^{\mathrm{d}}$ Four patients with missing data. 
ischemic stroke. The interrater agreement for the presence or absence of cSS was $89.6 \%$ (Cohen $\kappa=$ 0.79 ) and for cSS categories was $89.6 \%$ (weighted Cohen $\kappa=0.75)$. Forty-one patients $(34.7 \%$, 95\% confidence interval [CI]: $26.2 \%-44.6 \%)$ had cSS at baseline. The presence of cSS was strongly associated with a history of transient focal neurologic episodes ( $48.8 \%$ vs $8.8 \% ; p<0.001$, respectively) and the presence of $\geq 5 \mathrm{CMBs}(48.8 \%$ vs $27.3 \%$; $p=$ 0.019 , respectively) (table e-1 on the Neurology ${ }^{\circledR}$ Web site at www.neurology.org). CAA patients with cSS were slightly older compared to patients without siderosis (mean age; 95\% CI: 73.2; 70.4-76 years vs 70.3; 68.2-72.5 years, respectively; $p=0.111$ ).

During a median follow-up time of 24 months (interquartile range 9-44 months), 23 of 118 patients (19.5\%, 95\% CI: 12.8-27.8) experienced a symptomatic lobar ICH; 4 of these 23 patients experienced multiple $(>1)$ sequential symptomatic lobar ICH during follow-up. The characteristics of our cohort according to subsequent lobar ICH are shown in table 2. Fourteen of 23 patients (61\%) with lobar

Figure 1 Cortical superficial siderosis and ICH
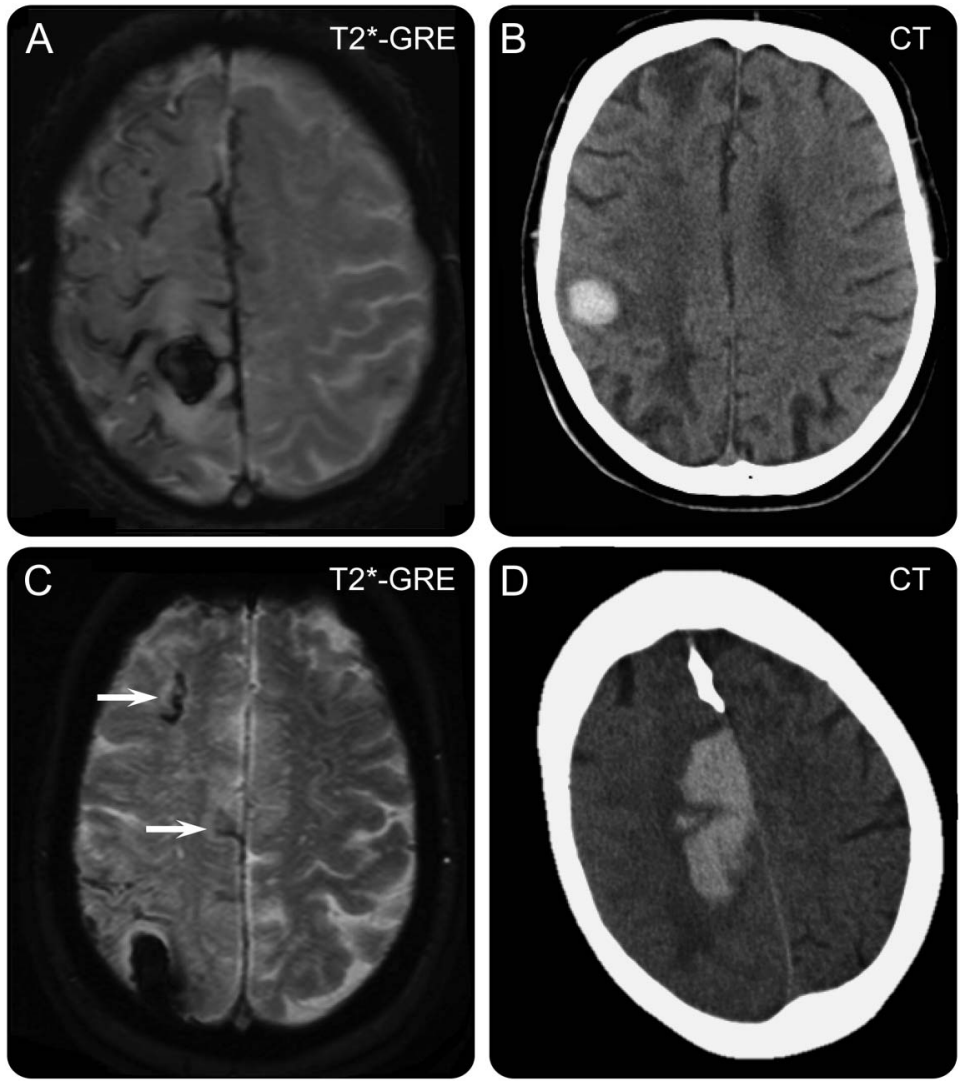

Subsequent intracerebral hemorrhage $(\mathrm{ICH})$ at (or close to) the site of preexisting siderosis in 2 patients with superficial siderosis. Female patient, aged 85 years; time interval between baseline imaging $(A)$ and incident $I C H(B): 19$ months. Male patient, aged 80 years; time interval between baseline imaging (C) and incident ICH (D): 35 months. (A, C) Baseline MRI, T2*-weighted images. (B, D) Follow-up unenhanced CT scans. T2*-GRE $=$ T2*-weighted gradient-recalled echo.
ICH during follow-up had cSS on baseline MRI: 3 had focal cSS and 11 had disseminated cSS (table 2). For 7 of these 14 patients, the subsequent ICH was anatomically correlated with the area of cSS (figure 1). Two of the 4 patients with multiple symptomatic lobar ICH at follow-up had disseminated cSS. One patient (without cSS at baseline) experienced a symptomatic deep (thalamic) ICH.

In the group of CAA patients without symptomatic lobar ICH at baseline $(n=14$; one patient with pathologically proven CAA, 12 patients with probable CAA, and one with possible CAA), 7 had cSS, which was disseminated in 5 cases. During a median follow-up time of 15.1 months (interquartile range 7.8-39.8), 3 of these patients experienced a symptomatic lobar ICH, all of whom had disseminated cSS at baseline scans.

In Kaplan-Meier analysis, the presence of cSS and disseminated cSS at baseline scans were predictors of time until ICH $(p=0.0045$ and $p=0.0009$, respectively, by the log-rank test) (figure 2). The risk of symptomatic lobar ICH at 4 years of follow-up was 25\% (95\% CI: 7.6\%-28.3\%) for patients without cSS at baseline, $28.9 \%$ (95\% CI: 7.7\%-76.7\%) for those with focal cSS, and 74\% (95\% CI: 44.1\%$95.7 \%$ ) for patients with disseminated cSS $(p=$ 0.0031 by the long-rank test for each category increase).

In univariate analysis, any cSS presence was a predictor of symptomatic lobar ICH (HR: 3.18; 95\% CI: $1.37-7.39 ; p=0.007)$, with an increased risk associated with disseminated cSS (HR: 4.07 compared with no siderosis; 95\% CI: 1.66-9.96; $p=$ $0.002)$. Focal cSS was associated with a nonsignificant increased hazard of subsequent ICH (HR: $1.91 \mathrm{com}-$ pared with no siderosis; 95\% CI: $0.51-7.19 ; p=$ 0.340 ). For each increase in category of cSS (i.e., from no siderosis, to focal, and to disseminated siderosis), the associated HR was 2.06 (95\% CI: 1.31-3.24; $p=$ 0.002 ). A history of symptomatic hemorrhage (before the index symptomatic ICH) (HR: $1.87 ; p=0.177$ ), presence of CMBs (HR: 2.17; $p=0.125$ ), and presence of $\geq 2$ or $\geq 5$ CMBs (HR: $1.55 ; p=0.318$ and HR: $1.33 ; p=0.507$, respectively) were not associated with future symptomatic lobar ICH in univariate analysis. Only age was associated with an increased hazard of subsequent ICH (HR: 1.07; $p=0.006$ ).

Prespecified multivariable Cox regression models demonstrated that cSS and disseminated cSS were independently associated with increased risk of symptomatic lobar ICH at follow-up, after adjusting for the presence of $\geq 2 \mathrm{CMBs}$ and age (table 3). These effect sizes remained consistent in similar multivariable models controlling for $\mathrm{CMB}$ number, $\mathrm{CMBs}$ presence, and $\geq 5$ CMBs. Our main results were also consistent in sensitivity analyses including cSS, 
Figure 2 Time to symptomatic lobar ICH during follow-up

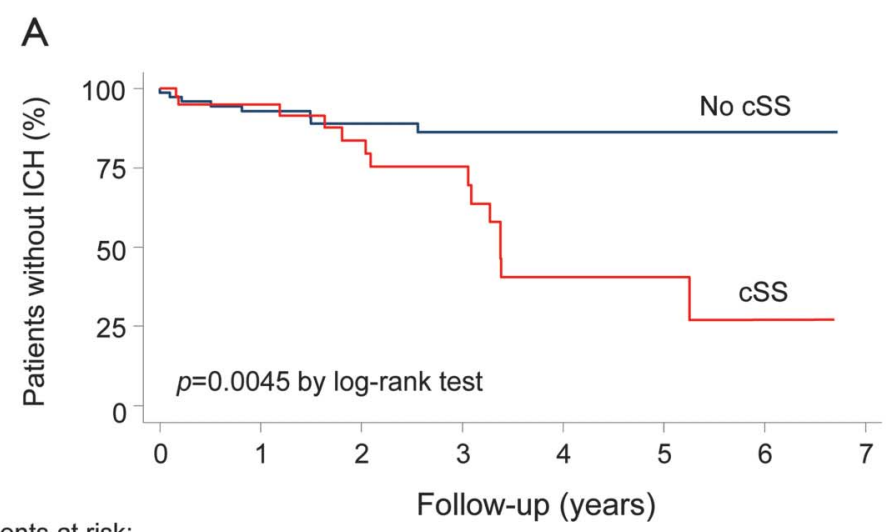

\begin{tabular}{|c|c|c|c|c|c|c|}
\hline & & & & 10 & & \\
\hline $\mathrm{No}$ siderosis 77 & & & & & & 7 \\
\hline Siderosis present 41 & 28 & 21 & 13 & 6 & 3 & 1 \\
\hline
\end{tabular}

\section{B}

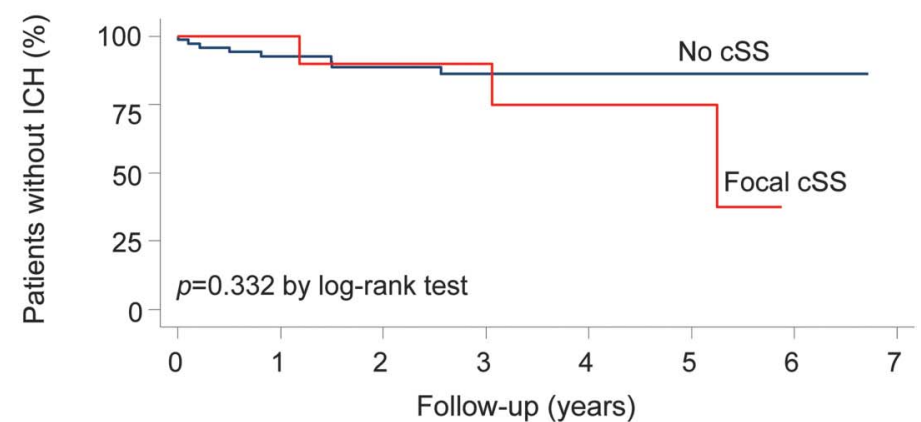

Patients at risk:

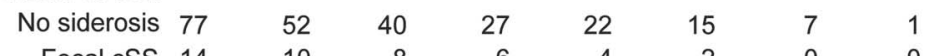
$\begin{array}{llllllll}\text { Focal cSS } & 14 & 10 & 8 & 6 & 4 & 2 & 0\end{array}$

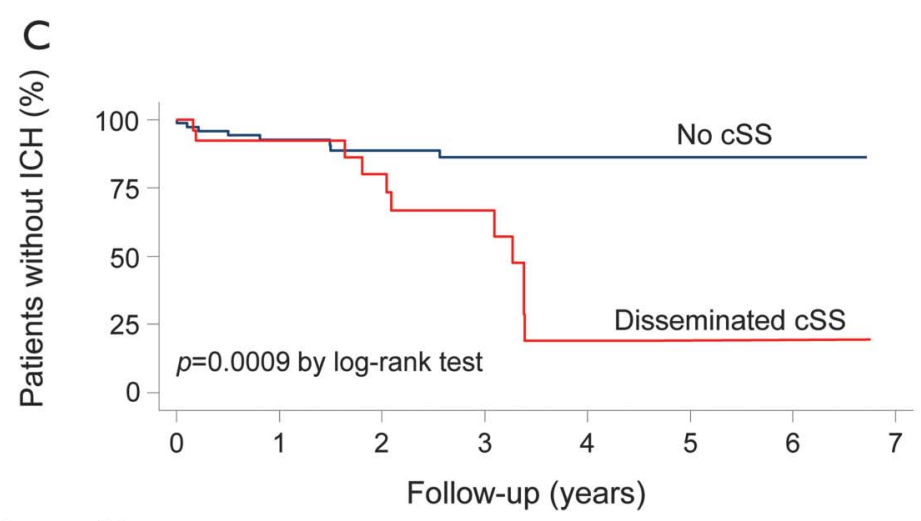

Patients at risk:

$\begin{array}{lllllllll}\text { No siderosis } & 77 & 52 & 40 & 27 & 22 & 15 & 7 & 1\end{array}$

$\begin{array}{llllllll}\text { Disseminated cSS } & 27 & 18 & 13 & 7 & 2 & 1 & 1\end{array}$

Kaplan-Meier estimates of progression to symptomatic lobar intracerebral hemorrhage (ICH) in the presence of (A) cortical superficial siderosis (cSS), (B) focal cSS, and (C) disseminated ( $>3$ sulci) cSS in all patients with cerebral amyloid angiopathy. Testing of significance is by the log-rank test.

CMBs, history of symptomatic ICH, and age in multivariable models (see table e-2).

All the results of Kaplan-Meier and prespecified Cox regression analyses remained consistent in sensitivity analyses, which included only CAA patients with symptomatic lobar ICH at baseline (figure e-1) and patients who had their MRI within 6 months of the index ICH (data not shown).

DISCUSSION In this multicenter retrospective cohort study, we found that cSS on T2*-GRE MRI (reflecting hemosiderin deposition in the subpial superficial cortical layers) significantly increases the risk of future symptomatic lobar ICH in patients with CAA. ${ }^{16}$ The risk rate for $\mathrm{ICH}$ was greatest for patients with disseminated cSS, involving multiple sulci on baseline scans. These results remained consistent after adjusting for age, the presence of multiple $(\geq 2)$ lobar CMBs (a hemorrhagic marker of CAA previously shown to influence the risk of ICH), ${ }^{12}$ and previous symptomatic ICH before the index inclusion event. In a subanalysis of those patients who presented with symptomatic ICH at baseline, cSS remained a predictor of $\mathrm{ICH}$ risk, consistent with the main analysis.

cSS is emerging as a common and characteristic feature of CAA.,24 One study reported cSS in $60.5 \%$ ( $\mathrm{n}=38$; mean age $70 \pm 6.4$ years $)$ of patients with histopathologically proven CAA, compared with no control subjects with histopathologically proven non-CAA ICH $\left(\mathrm{n}=22\right.$; mean age $54 \pm 18$ years). ${ }^{13}$ Another recent study found cSS in $40 \%$ of patients with a clinic-radiologic diagnosis of probable CAArelated $\mathrm{ICH}$, but only $5 \%$ of patients with purely deep $\mathrm{ICH}$, presumed to be due to hypertensive arteriopathy. ${ }^{25}$ Increasing data support the hypothesis that cSS might precede lobar ICH in patients with CAA. ${ }^{15,26-28}$ In a recent retrospective study, 51 patients with cSS and no apparent cause other than CAA were identified through a single-center systematic database search and followed up for a median of 35.3 months. ${ }^{15}$ Over this period, 24 patients (47.1\%) experienced any new "intracranial hemorrhage" (ICH or acute convexity subarachnoid hemorrhage): 18 patients (35.3\%) had an ICH, of which 13 were at the site of preexisting siderosis. ${ }^{15}$ This study was limited by the incomplete ascertainment of outcome intracranial bleeding events (without details of how many were symptomatic), and the lack of a comparison group without cSS at baseline.

Our larger study confirms that cSS is a risk factor for future symptomatic ICH in CAA, independent of multiple lobar CMBs. It is hypothesized that repeated episodes of hemorrhage from brittle superficial cortical or leptomeningeal CAA-affected vessels into the subarachnoid space leads to subpial hemosiderin deposition and cSS on MRI. ${ }^{29}$ The finding of cSS without previous $\mathrm{ICH}$, and its tendency to occur distantly from previous ICH, favor this "primary" mechanism, ${ }^{13,15}$ rather than leakage of blood into the subarachnoid space secondary to previous lobar "macro" ICH. ${ }^{25}$ Consequently, cSS may be a marker of increased cortical and leptomeningeal small-vessel 


\begin{tabular}{|llr|}
\hline Table $3 \quad \begin{array}{l}\text { Prespecified multivariate analyses of predictors of symptomatic lobar } \\
\text { intracerebral hemorrhage during follow-up in patients with cerebral } \\
\text { amyloid angiopathy }\end{array}$ & $p$ Value \\
Variables & HR (95\% Cl) & $0.040^{\mathrm{a}}$ \\
Model 1 & $2.53(1.05-6.15)$ & 0.836 \\
Presence of cortical superficial siderosis & $1.10(0.45-2.71)$ & $0.028^{\mathrm{a}}$ \\
Presence of $\geq 2$ CMBs & $1.06(1.01-1.11)$ & \\
Age (for each year increase) & $3.16(1.35-7.43)$ & $0.008^{\mathrm{b}}$ \\
Model 2 & $1.07(0.44-2.63)$ & 0.876 \\
Disseminated cortical superficial siderosis & $1.07(1.01-1.13)$ & $0.017^{\mathrm{a}}$ \\
Presence of $\geq 2$ CMBs & & \\
Age (for each year increase) &
\end{tabular}

Abbreviations: $\mathrm{Cl}$ = confidence interval; $\mathrm{CMB}$ = cerebral microbleed; $\mathrm{HR}=$ hazard ratio. The models remain consistent if number of $\mathrm{CMBs}$, presence of $\mathrm{CMBs}$, or $\geq 5 \mathrm{CMBs}$ are included.

a Statistically significant difference at $p<0.05$.

${ }^{b}$ Statistically significant difference at $p<0.01$.

fragility, high CAA disease activity, and vulnerability to subarachnoid bleeding, which in some circumstances may extend and develop into a lobar $\mathrm{ICH}{ }^{30}$ Indeed, a neuropathologic series of 6 autopsy cases of subcortical hematoma caused by CAA showed that multiple leptomeningeal arteries can rupture into the subarachnoid space and the brain parenchyma. ${ }^{30}$ This hypothesis is supported by the observation that symptomatic ICH has been noted at (or close to) the site of previous siderosis, ${ }^{15}$ although we found that $\mathrm{ICH}$ only occurred at the site of cSS in $50 \%$ of cases. We found the highest ICH risk in patients with disseminated cSS, which may indicate widespread and numerous leptomeningeal vessels damaged by advanced CAA, providing multiple potential initiation sites for future $\mathrm{ICH}$, increasing the probability of this outcome. ${ }^{30}$ Further serial MRI studies will help to unravel the sequence of events and mechanisms linking CAA, cSS, and lobar ICH, including asymptomatic bleeding. ${ }^{31}$ Assessment of the associations between $A P O E$ genotype (which was not available in our cohort) and the extent or severity of CAArelated pathology in leptomeningeal vessels may also be of interest.

Although our results suggest an increased risk of subsequent ICH in CAA patients with cSS on MRI, antithrombotic drug use probably also has a role in this risk by impairing hemostatic mechanisms. ${ }^{2,9,12}$ In our retrospective cohort, it was not possible to systematically collect data on the use of antithrombotic drugs. However, routine clinical practice in all 4 centers in our study was to avoid all antiplatelet agents (including aspirin) and anticoagulants in patients with suspected CAA, unless there was a very strong indication, so is unlikely to have contributed significantly to the outcome events in our study.
Our findings might have important implications for patients presenting with transient focal neurologic episodes (sometimes called "amyloid spells"32) resembling TIAs, migraine auras, or focal seizures, ${ }^{32-34}$ which are increasingly recognized in CAA. It was recently reported that such attacks are often related to CSS and herald a high early risk of symptomatic lobar ICH $(24.5 \%$ [95\% CI: $15.8 \%-36.9 \%$ ] at 8 weeks). ${ }^{33}$ Thus, these types of attacks (which are often recurrent and stereotyped) should alert the clinician to the possibility of cSS; giving antithrombotic drugs to these patients because of misdiagnosis as TIA could significantly increase the risk of serious future ICH. ${ }^{31}$ Moreover, our current data suggest that disseminated siderosis may be a particular risk factor for future ICH in this situation.

Perhaps surprisingly, we did not find a significant association between multiple lobar $\mathrm{CMBs}$ and future ICH risk, ${ }^{11,12}$ which may reflect selection bias toward generally advanced disease with high prevalence of multiple lobar CMBs in our cohort. Although our study had adequate power to detect an increase in the risk of $\mathrm{ICH}$ in the presence of cSS, our sample size was not large enough to investigate additional potential baseline predictors of ICH (e.g., index ICH volume, APOE genotype) or to investigate ICH risk in the subset of patients without lobar ICH at baseline. Other potential limitations include the retrospective design, and the potential of bias in our sample because the requirement for MRI may exclude more severe cases of ICH. Furthermore, a proportion of otherwise eligible patients did not have reliable follow-up data. Finally, we did not have pathologic confirmation of CAA pathology, and acknowledge that the Boston criteria have imperfect specificity, especially for the "possible CAA" category. ${ }^{16,35}$

Our findings nevertheless suggest that cSS is a useful independent prognostic marker of intracerebral bleeding risk in CAA. Larger cohorts are needed to confirm our results and explore the potential implications for CAA treatment (e.g., avoiding antithrombotic agents in patients with disseminated cSS). cSS could also have implications for future disease-modifying treatments in CAA that may cause amyloid- $\beta$ shifts between brain parenchyma and blood vessels (e.g., immunotherapy); CMBs have been considered a possible caution for such treatments, ${ }^{36}$ but the role of cSS in this setting remains to be determined.

\section{AUTHOR CONTRIBUTIONS}

Statistical analysis was conducted by A. Charidimou and Z. Fox. A. Charidimou: project design, data collection, data analysis, write-up. Z. Fox: data analysis, data interpretation. A. Peeters, Y. Vandermeeren, P. Laloux, and J.-C. Baron: data collection, critical revisions. R. Jäger: imaging analysis advice, critical revisions. D.J. Werring: project design, supervision, data interpretation, write-up, obtaining funding.

\section{ACKNOWLEDGMENT}

The authors thank Dr. Simone Gregoire for her assistance with establishing the multicenter cohort and for technical assistance with database organization. 


\section{STUDY FUNDING}

No targeted funding reported.

\section{DISCLOSURE}

A. Charidimou receives research support from the Greek State Scholarship Foundation, the Stroke Association, and the British Heart Foundation. A. Peeters, R. Jäger, Z. Fox, Y. Vandermeeren, P. Laloux, and J.-C. Baron report no disclosures. D. Werring receives research support from the Department of Health/Higher Education Funding Council for England (Clinical Senior Lectureship Award), the Stroke Association, and the British Heart Foundation. Part of this work was undertaken at UCLH/ UCL who received a proportion of funding from the Department of Health's NIHR Biomedical Research Centres funding scheme. Go to Neurology.org for full disclosures.

Received April 2, 2013. Accepted in final form August 6, 2013.

\section{REFERENCES}

1. MRC CFAS. Pathological correlates of late-onset dementia in a multicentre, community-based population in England and Wales. Neuropathology Group of the Medical Research Council Cognitive Function and Ageing Study (MRC CFAS). Lancet 2001;357:169-175.

2. Charidimou A, Gang Q, Werring DJ. Sporadic cerebral amyloid angiopathy revisited: recent insights into pathophysiology and clinical spectrum. J Neurol Neurosurg Psychiatry 2012;83:124-137.

3. Viswanathan A, Greenberg SM. Cerebral amyloid angiopathy in the elderly. Ann Neurol 2011;70:871-880.

4. Vinters HV. Cerebral amyloid angiopathy: a critical review. Stroke 1987;18:311-324.

5. Bejot Y, Cordonnier C, Durier J, Aboa-Eboule C, Rouaud O, Giroud M. Intracerebral haemorrhage profiles are changing: results from the Dijon population-based study. Brain 2013;136:658-664.

6. Morgenstern LB, Hemphill JC III, Anderson C, et al. Guidelines for the management of spontaneous intracerebral hemorrhage: a guideline for healthcare professionals from the American Heart Association/American Stroke Association. Stroke 2010;41:2108-2129.

7. Vermeer SE, Algra A, Franke CL, Koudstaal PJ, Rinkel GJ. Long-term prognosis after recovery from primary intracerebral hemorrhage. Neurology 2002;59:205-209.

8. Bailey RD, Hart RG, Benavente O, Pearce LA. Recurrent brain hemorrhage is more frequent than ischemic stroke after intracranial hemorrhage. Neurology 2001;56:773-777.

9. Viswanathan A, Rakich SM, Engel C, et al. Antiplatelet use after intracerebral hemorrhage. Neurology 2006;66: 206-209.

10. O'Donnell HC, Rosand J, Knudsen KA, et al. Apolipoprotein $\mathrm{E}$ genotype and the risk of recurrent lobar intracerebral hemorrhage. N Engl J Med 2000;342:240-245.

11. Greenberg SM, Eng JA, Ning M, Smith EE, Rosand J. Hemorrhage burden predicts recurrent intracerebral hemorrhage after lobar hemorrhage. Stroke 2004;35:1415-1420.

12. Biffi A, Halpin A, Towfighi A, et al. Aspirin and recurrent intracerebral hemorrhage in cerebral amyloid angiopathy. Neurology 2010;75:693-698.

13. Linn J, Halpin A, Demaerel P, et al. Prevalence of superficial siderosis in patients with cerebral amyloid angiopathy. Neurology 2010;74:1346-1350.

14. Linn J, Herms J, Dichgans M, et al. Subarachnoid hemosiderosis and superficial cortical hemosiderosis in cerebral amyloid angiopathy. AJNR Am J Neuroradiol 2008;29: $184-186$.
15. Linn J, Wollenweber FA, Lummel N, et al. Superficial siderosis is a warning sign for future intracranial hemorrhage. J Neurol 2012;260:176-181.

16. Knudsen KA, Rosand J, Karluk D, Greenberg SM. Clinical diagnosis of cerebral amyloid angiopathy: validation of the Boston criteria. Neurology 2001;56: 537-539.

17. Gregoire SM, Chaudhary UJ, Brown MM, et al. The Microbleed Anatomical Rating Scale (MARS): reliability of a tool to map brain microbleeds. Neurology 2009;73: 1759-1766.

18. Greenberg SM, Nandigam RN, Delgado P, et al. Microbleeds versus macrobleeds: evidence for distinct entities. Stroke 2009;40:2382-2386.

19. Inzitari D, Simoni M, Pracucci G, et al. Risk of rapid global functional decline in elderly patients with severe cerebral age-related white matter changes: the LADIS study. Arch Intern Med 2007;167:81-88.

20. Kidwell CS, Greenberg SM. Red meets white: do microbleeds link hemorrhagic and ischemic cerebrovascular disease? Neurology 2009;73:1614-1615.

21. Concato J, Peduzzi P, Holford TR, Feinstein AR. Importance of events per independent variable in proportional hazards analysis. I. Background, goals, and general strategy. J Clin Epidemiol 1995;48:1495-1501.

22. Peduzzi P, Concato J, Feinstein AR, Holford TR. Importance of events per independent variable in proportional hazards regression analysis. II. Accuracy and precision of regression estimates. J Clin Epidemiol 1995;48:1503-1510.

23. von Elm E, Altman DG, Egger M, Pocock SJ, Gotzsche PC, Vandenbroucke JP. The Strengthening the Reporting of Observational Studies in Epidemiology (STROBE) statement: guidelines for reporting observational studies. Lancet 2007;370:1453-1457.

24. Vernooij MW, Ikram MA, Hofman A, Krestin GP, Breteler MM, van der Lugt A. Superficial siderosis in the general population. Neurology 2009;73:202-205.

25. Charidimou A, Jager HR, Fox Z, et al. Prevalence and mechanisms of cortical superficial siderosis in cerebral amyloid angiopathy. Neurology 2013;81:626-632.

26. Kumar S, Goddeau RP, Selim MH, et al. Atraumatic convexal subarachnoid hemorrhage: clinical presentation, imaging patterns, and etiologies. Neurology 2010;74: 893-899.

27. Katoh M, Yoshino M, Asaoka K, et al. A restricted subarachnoid hemorrhage in the cortical sulcus in cerebral amyloid angiopathy: could it be a warning sign? Surg Neurol 2007;68:457-460.

28. Kawahara I, Nakamoto M, Matsuo Y, Tokunaga Y. A case of cerebral amyloid angiopathy in which a restricted subarachnoid hemorrhage recurred in the cortical sulcus following a subcortical hemorrhage [in Japanese]. No Shinkei Geka 2010;38:551-555.

29. Koeppen AH, Dickson AC, Chu RC, Thach RE. The pathogenesis of superficial siderosis of the central nervous system. Ann Neurol 1993;34:646-653.

30. Takeda S, Yamazaki K, Miyakawa T, et al. Subcortical hematoma caused by cerebral amyloid angiopathy: does the first evidence of hemorrhage occur in the subarachnoid space? Neuropathology 2003;23:254-261.

31. Charidimou A, Baron JC, Werring DJ. Transient focal neurological episodes, cerebral amyloid angiopathy, and 
intracerebral hemorrhage risk: looking beyond TIAs. Int J Stroke 2013;8:105-108.

32. Charidimou A, Law R, Werring DJ. Amyloid "spells" trouble. Lancet 2012;380:1620.

33. Charidimou A, Peeters A, Fox Z, et al. Spectrum of transient focal neurological episodes in cerebral amyloid angiopathy: multicentre magnetic resonance imaging cohort study and meta-analysis. Stroke 2012;43: 2324-2330.

34. Greenberg SM, Vonsattel JP, Stakes JW, Gruber M, Finklestein SP. The clinical spectrum of cerebral amyloid angiopathy: presentations without lobar hemorrhage. Neurology 1993;43:2073-2079.

35. van Rooden $S$, van der Grond J, van den Boom R, et al. Descriptive analysis of the Boston criteria applied to a Dutch-type cerebral amyloid angiopathy population. Stroke 2009;40:3022-3027.

36. Sperling RA, Jack CR Jr, Black SE, et al. Amyloid-related imaging abnormalities in amyloid-modifying therapeutic trials: recommendations from the Alzheimer's Association Research Roundtable Workgroup. Alzheimers Dement 2011;7:367-385.

\section{Guide the Future of Neurology-Become a Mentor!}

The Academy's Neurology Career Center is working to bring experienced members together with members who seek guidance on their career path. AAN Mentor Connect needs volunteer Mentors who are willing to share their expertise, insights, and experiences with Mentees.

This flexible program, available only to AAN members, matches prospective Mentors and Mentees, and enables you to develop a plan with the Mentee that has a mutually agreeable schedule and expectations.

Enjoy the personal satisfaction of making a valued contribution to the career of a fellow AAN member. Visit www.aan.com/view/Mentor to learn more and register to be a Mentor today.

\section{Earn 20 CME Credits Toward MOC with New NeuroPI ${ }^{\mathrm{SM}}$ Modules}

Choose from the latest lineup of quality modules to join the AAN's exclusive performance improvement programs designed to help you address both the Performance in Practice (PIP) and Continuing Medical Education (CME) components of Maintenance of Certification (MOC).

- NEW! Distal Symmetric Polyneuropathy (DSP) includes eight quality measures, addressing accurate and appropriate evaluation/monitoring of DSP and associated symptoms to guide treatment options, patient safety, and best practices to assist patients in managing their pain and improving quality of life

- Acute Stroke addresses six quality measures, including deep vein thrombosis prophylaxis (DVT) for ischemic stroke or intracranial hemorrhage, discharged on antiplatelet therapy, dysphagia screening, rehabilitation service considerations, and more

- Dementia includes 10 quality measures addressing underuse of effective services and patientcentered care strategies, and patient safety issues

Learn about all of the other available modules and purchase yours today: www.aan.com/view/neuropi 